\title{
Massive arterial bleeding from a single rectal vessel
}

\author{
JD Harrison, A Calatayud, VR Thava, RM Kirby
}

\begin{abstract}
Summary
We report a case of massive rectal haemorrhage arising from a single ectatic arterial vessel above the haemorrhoidal cushion in normal rectal mucosa. Use of an anal retractor enabled identification of the bleeding vessel and avoided a major laparatomy.
\end{abstract}

Keywords: rectal bleeding

Massive fresh rectal bleeding in the elderly can be difficult to diagnose and manage. Whilst lower gastrointestinal bleeding often settles spontaneously, a proportion of patients continue to bleed and require radiological or surgical intervention.

Lower gastrointestinal haemorrhage is commonly due to diverticular disease, neoplasia or angiodysplasia, ${ }^{1}$ and localisation of the bleeding can be a formidable task. If the site of haemorrhage is unknown even after angiography $^{2}$ or scintigraphy, ${ }^{3}$ then harzardous 'blind' major surgery may be mandatory.

\section{Case report}

A 68-year-old man receiving radical radiotherapy for a carcinoma of the tongue presented as an emergency to the on-call medical team with what was initially described as melaena. There was no history of upper gastrointestinal symptoms or nonsteroidal anti-inflammatory agent ingestion. An upper gastrointestinal endoscopy showed distal gastritis only, with no bleeding lesion seen. His vital signs were stable with a haemoglobin of $12 \mathrm{~g} / \mathrm{dl}$.

He developed fresh rectal bleeding requiring blood transfusion and was referred for a surgical opinion. At this time there was no abdominal abnormality and rectal examination was unremarkable apart from the presence of fresh blood. Under local anaesthesia selective mesenteric angiography was performed placing a $5 \mathrm{~F}$ sidewinder catheter selectively into the inferior and superior mesenteric arteries. Access was gained via the right transfemoral route, injecting Ultravist 370 (Iopromide $0.769 \mathrm{~g} / \mathrm{ml}$, Schering AG) with a Medrad injector. No abnormality was seen in the territory of the superior mesenteric artery other than a few short atheromatous segments. The inferior mesenteric artery angiogram showed a tortuous and ectatic distal segment of the superior haemorrhoidal artery with contrast emerging from it and pooling within the rectum (figure 1).
Examination under anaesthesia was carried out in the operating theatre. Repeat digital rectal examination was normal and sigmoidoscopy was unhelpful as large amounts of liquid blood obscured the view. After insertion of a Parks anal retractor, a single arterial spurting vessel was seen well above the haemorrhoidal cushion in otherwise normal mucosa. The bleeding vessel was suture ligated with three 2/0 Vicryl sutures (Ethicon Corporation, Edinburgh, UK). Post-operatively he had an uneventful recovery. He returned to the oncology ward five days later and was subsequently discharged home.

\section{Discussion}

While the majority of elderly patients with rectal bleeding settle with conservative management, a small proportion continue to bleed from a multiplicity of different lesions, particularly diverticular disease, neoplastic and angiodysplastic lesions. The presentation of arterial bleeding from a single abnormal vessel in otherwise normal mucosa has not been described previously, although there is a comparable condition in the stomach. This is the 'Dieulafoy lesion' which consists of an abnormal bleeding vessel in normal gastric mucosa which can be responsible for massive and occasionally fatal upper gastrointestinal bleeding. In a recent study this lesion (of unknown aetiology) accounted for $6 / 1000$ patients with upper gastrointestinal bleeding. ${ }^{4}$

A recent article suggested the use of visceral angiography before surgery in patients over 45 years of age after laparotomy when no cause has been found. Exploratory laparotomy was

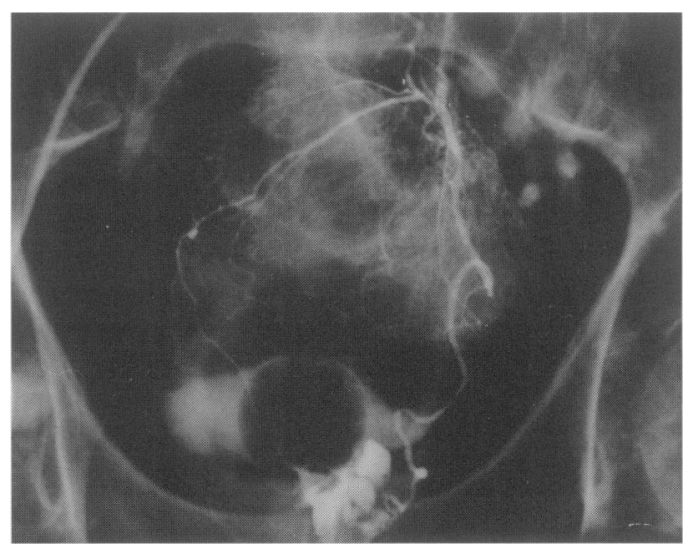

Figure Selective inferior mesenteric angiogram showing the ectatic distal superior haemorrhoidal artery with contrast medium in the rectum (bottom right) 


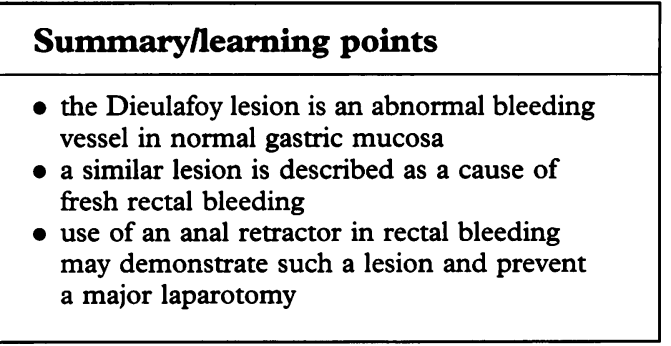

recommended at an early stage for younger patients, and for older patients after nondiagnostic angiography (62 of 131 patients had a negative angiogram). ${ }^{2}$ Other studies have shown that the incidence of angiodysplasia and diverticular disease is approximately equal as a cause of massive lower gastrointestinal bleeding in the elderly. The majority of angiodysplastic lesions are right-sided ${ }^{5-7}$ and bleeding from diverticula is as likely to be from

1 Athanasoulis CA, Galdabini J, Waltman AC, Novelline RA, Greenfield AJ, Ezpeleta ML. Angiodysplasia of the colon: a cause of rectal bleeding. Cardiovasc Radiol 1977; 1: 3-13.

2 Thompson JN, Salem RR, Hemingway AP, et al. Specialist investigation of obscure gastrointestinal bleeding. Gut 1987; 28: $47-51$.

3 Winzelberg GG, McKusick KA, Froelich JW, Callahan RJ, Strauss HW. Detection of gastrointestinal bleeding with 99mTc-labeled red blood. Semin Nucl Med 1982; 12: 13999m.

4 Arora A, Mehrotra R, Patnaik PK, Pande G, Ahlawat S, Bhargava DK. Dieulafoy's lesion: a rare cause of massive upper gastrointestinal bleeding. Trop Gastroenterol 1991; 12: $25-30$.

5 Boley S, DiBiase A, Brandt L, Sammartano R. Lower intestinal bleeding in the elderly. Am $\mathcal{F}$ Surg $1979 ; 137$ : 57 64.

6 Casarella WJ, Galloway SJ, Taxin RN, Follett DA, Pollock

EJ, Seaman WB. 'Lower' gastrointestinal tract hemorrhage: new concepts based on arteriography. $A f R$ 1974; 121: 357 68. the right side as the left. ${ }^{8-11}$ It has therefore been suggested that a blind right hemicolectomy should be the segmental procedure of choice in massive bleeding of unknown aetiology. ${ }^{12}$ Others have suggested more aggressive surgery because of the high re-bleeding rate (in the region of $65 \%$ ) after blind segmental resection. ${ }^{13}$ Although these studies show that such procedures can be performed with an acceptable mortality rate, clearly an actively bleeding rectal lesion will be inadequately treated by either of these recommended options, in addition to exposing the patients to the hazards of a major operation in an unstable condition.

We would suggest that, in patients requiring surgery as a result of a fresh rectal bleeding, a thorough rectal examination with an anal retractor rather than a sigmoidoscope is mandatory, since it may spare the patient from an unnecessary major laparotomy.

7 Miller K, Tutton R, Bell K, Simon B. Angiodysplasia of the colon. Radiology 1979; 132: 209-13.

8 Earley C. The management of massive hemorrhage from diverticular disease of the colon. Surg Gynecol Obstet 1959; 108: 49.

9 Klein $\mathbf{R}$, Gallagher D. Massive colonic bleeding from diverticular disease. Am f Surg 1969; 118: 553.

$10 \mathrm{McGuire} \mathrm{H}$, Haynes B. Massive hemorrhage from diverticulosis of the colon: guidelines for therapy based on bleeding patterns observed in 50 cases. Ann Surg 1972; 178: 847 .

11 Quinn W. Gross hemorrhage from presumed gross diverticular disease of the colon: results of treatment in 103 cular disease of the colon: result
patients. Ann Surg 1961; 153: 851 .

12 Brasfield R. Empiric right colectomy for massive gastrointestinal bleeding. Arch Surg 1961; 83: 247.

13 Drapanas T, Pennington G, Kappelman M, Lindsey ES. Emergency subtotal colectomy: preferred approach to management of massively bleeding diverticular disease. Ann Surg 1973; 177: 519-26.

Sigmund Freud (1856-1939) was born in Freiburg, Moravia, son of a wool merchant. He studied medicine in Vienna and became a neurologist at Vienna General Hospital. He studied with Charcot in Paris and became a dedicated psychiatrist with a reputation for the management of hysteria, infantile sexuality, the interpretation of dreams, and eventually Freudian psychoanalysis. As a result of Nazi oppression, he fled to London. He died in his home in Hampstead which is now a museum to his memory.

In 1884 he showed the value of cocaine as a local anaesthetic in ophthalmology. This fact led to Halsted's addiction to drugs. - DG fames 